Original Research Paper

\title{
Apoptosis Inducer Capacity of Cardiotonic Steroids of Urginea maritima Extract on SH-SY5Y Neuroblastoma Cells, with Less Susceptibility among Neuron-Module Cells
}

\author{
${ }^{1,2}$ Maati M. Elghuol, ${ }^{1}$ Khalilah Abdul Khalil, \\ ${ }^{2}$ Mazatulikhma Mat Zain and ${ }^{1}$ Mohamed Saifulaman Mohamed Said \\ ${ }^{1}$ Biomolecular Science, Faculty of Applied Science, University Technology MARA (UiTM), 40450 Shah Alam, Malaysia \\ ${ }^{2}$ Tissue Culture Research Laboratory, Institute of Science (IOS), University Technology MARA, 40450 Shah Alam, Malaysia
}

Article history
Received: 07-04-2016
Revised: 24-05-2016
Accepted: 26-05-2016
Corresponding Author:
Mohamed Saifulaman
Mohamed Said
Biomolecular Science, Faculty
of Applied Science, University
Technology MARA (UiTM),
40450 Shah Alam, Malaysia
Email:drsaifulamann@yahoo.com
or
Email:maati007guoluitm@gmail.com

\section{Introduction}

Neuroblastoma is the most common extra-cranial solid tumor that mainly affects infants. Approximately $10-15 \%$ of most deadly malignancies of all predicates are associated with neuroblastoma (Cernaianu et al., 2008; Oselkin et al., 2010). It is an extreme heterogeneity tumor, whereas high-risk Diseases are very aggressive metastatic tumors. Although the most

\begin{abstract}
Natural products have played a significant role in conventional treatment and in the development of new drugs for a wide range of illnesses, including malignant disease. Urginea maritima is classified as an herb is endemic to the Mediterranean area. This herb was renowned in traditional ethnopharmacological applications. Notwithstanding, there is a lack of evidence pertaining to the characterization of the Urginea maritima constituents contained within a water-based extract and their bioactivity in human neurological disorders. Therefore, we sought to investigate the composition and to evaluate its antitumour capability against the human malignant neuroblastoma cells. Through the utilization of several in vitro techniques, the present work illustrates that cardiotonic steroids are the main constituents of Urginea maritima aqueous extract and grouping of polyphenolic compounds are present. This result was based on interpretation of the mass spectrum obtained via electrospray ionization timeof-flight tandem mass spectrometry. The current study finding confirms that the constituents of the extract trigger a series of toxic reactions. In terms of cell proliferation inhibition, malignant SH-SY5Y neuroblastoma cell proliferation was selectively inhibited in a time- dose dependent manner. As verified quantitatively using the MTS colorimetric assay, with less toxicity was observed toward neuron-model cells. Apoptosis was efficiently induced in SH-SY5Y neuroblastoma cells as evidenced by Fluorescent microscopy using PI staining and DNA-specific dye DAPI revealed nuclear fragmentation, which profounds the apoptosis mechanism. Our novel findings clearly emphasize that the principal constituents of this unusual natural product can exhibit dual effects on neuron cells. This preferential selectivity provides an interesting basis for widespread medical application and a promising therapeutic strategy against neurological diseases, specifically human malignant neuroblastoma disorders.
\end{abstract}

Keywords: Urginea maritima, Cardiotonic Steroids, Proliferation Inhibition, Neurotoxicity, Nuclear Fragmentation intensive multimodal therapies available are currently being applied, the survival rates of patients with highrisk neuroblastoma remain low. Thus, the identification of new pharmaceutical candidates for neuroblastoma malignancies is strongly needed (Park et al., 2010).

Extensive research has conducted over the last decade, in expanding knowledge concerning the pathogenesis mechanisms of neurological disorders are complex with many targets and pathways (Blaylock and Maroon, 2012). 
In line with these findings, natural products with their great structural diversity have offered, major opportunities for the identification of new suitable-drugs, which hold much promise as therapeutic agents to combat neurological diseases (Dunn et al., 2011).

$U$. maritima is renowned in traditional ethno pharmacological applications as far back as the time of the ancient Egyptians. Nevertheless, the anticancer biological activities of this medicinal plant are still largely unexplored (El-Seedi et al., 2013). Previous phytochemical analyses have identified Cardiotonic steroids as the major constituents in organic solvent extracts of U. maritima species (Kopp et al., 1996). Anthocyanins, flavonoids, fatty acids, polysaccharides and calcium oxalates are similarly present (AL-Tardeh and Delivopouos 2006; Huang et al., 2010). However, such knowledge of characterization and bioactivities properties regarding of $U$. maritima constituents of water-based extracts are poorly acknowledged. In this study, a more selective and a sensitivity analysis was designed by means of High-Performance Liquid Chromatography (HPLC) coupled with electrospray ionization time-of-flight tandem mass spectrometry (ESI-TOF-MS/MS) to explore the composition of phytochemical compounds in U. maritima water-based extracts. These analysis tools have been proven effective in identifying and quantifying the important pharmaceutical information of herbal medicines (Wang et al., 2000). Recent works have scientifically proven the efficiency and unique character of (CTS; Cardiotonic steroids) in targeting and attacking various cancer cells with less toxicity to non-malignant cells (Newman et al., 2008; Prassas and Diamandis, 2008). Noteworthy, compelling scientific evidence detected that malignant cells have exposure to cardiac steroids exhibit alterations in the activity of the $\mathrm{Na}+/ \mathrm{K}+$-ATPase (Yu, 2003; Mijatovic and Kiss, 2013). Thus, this class of compounds has received much attention as regards their potential as antineoplastic agents. Furthermore, in vitro and in vivo epidemiological data suggested that plant-derived cardiac steroids mediated anticancer activities through a regulated multitude of cellular processes such as proliferation, apoptosis and cell cycle arrest in various types of cancer cell lines, including human MCF-7, MDA-MB2311, prostate, melanoma, hepatoma cell, pancreatic, lung, colon cancer cells (Felth et al., 2009) and leukemia cell lines (Jiang et al., 2010; Waheed et al., 2012; Zhitu et al., 2012). Moreover, systematic works of the past few years, have discovered that polyphenol compounds have been playing an important role in protecting nerve cells from oxidative damage. Therefore; they may have valuable health effects and can be considered as therapeutic agents against neurological disorders (Jiang et al., 2010; Kelsey et al., 2011). In parallel of this evidence, the presence of grouping of polyphenolic compounds in the composition of $U$. maritima aqueous extract could be attributed to its efficient anti-tumorspecific action against neuroblastoma SH-SY5Y cell line. With displayed less toxicity in a direction to neuron-model cells. To the best of our knowledge, no data have published on anti-proliferation and apoptosis-inducing mechanisms underlying the effects of $U$. maritima ingredients against solid tumors (Blaylock and Maroon, 2012; Huang et al., 2010). Consequently, this study aims to investigate the phytochemical composition of $U$. maritima aqueous extracts and evaluates its antiproliferation and apoptosis-inducing effects against $\mathrm{SH}-$ SY5Y neuroblastoma cell lines. With hope to identify a new potent candidate, that would be feasible for the management of neuroblastoma disorders.

\section{Materials and Methods}

\section{Reagents and Chemicals}

CellTiter 96*AQueous Non-Radioactive Cell Proliferation Assay Solution (MTS; Promega, USA); Minimum Essential Medium Eagle (EMEM; Sigma, USA); Ham's F12 (Sigma, USA); non-essential amino acids $(100 \times)$ (PAA Laboratories $\mathrm{GmbH}$, Austria); Lglutamine (200 mM) (Sigma, USA); gentamicin $(10 \mathrm{mg}$ $\mathrm{mL}^{-1}$ ) (PAA Laboratories GmbH, Austria); Fetal Bovine Serum (FBS) (PAA Laboratories $\mathrm{GmbH}$, Austria); dimethyl sulfoxide (DMSO; Sigma, USA); Retinoic Acid (RA; Sigma, USA); Proscillaridin-A, powder (SigmaAldrich); Propidium Iodide (PI) stain (BD Pharmingen, USA); DAPI stain (Thermo Scientific Inc., USA).

\section{Aqueous Extract of U. maritima Preparation}

Bulbs of the medicinal plant $U$. maritima were collected from green mountains towns (Sosa Township) in Libya. The field studies did not involve any endangered/protected species. Prof. Mohammed Alsharif performed the botanical identification. The specimens were deposited in the herbarium section of the department of Botany Science, University of Garyounis, Libya. The dry bulbs of U. maritima were ground into a fine powder. A total of $100 \mathrm{~g}$ of this powder was weighed and mixed with $1000 \mathrm{~mL}$ boiling sterile double-distilled water. The mixture was shaken on a horizontal shaker at $37^{\circ} \mathrm{C}$ and $250 \mathrm{rpm}$ for $72 \mathrm{~h}$. Eventually, all of the supernatants was separated out and a $500 \mathrm{~mL}$ bottle-top filter (sterile, $0.22 \mu \mathrm{m}$ pores; Corning, USA) was used to obtain a highly purified, light brown filtrate. The filtered solution of $U$. maritima aqueous extract was lyophilized under sterile conditions in the freeze-dryer system. The concentrated U. maritima extract was dissolved in DMSO (Sigma, USA) to obtain a stock solution (the percentage of 
DMSO in the experiment did not exceed $0.5 \%$ ). Dilutions of the stock solution at $1.0 \mathrm{mg} \mathrm{mL}^{-1}$ were prepared, these solutions were stored at $4^{\circ} \mathrm{C}$.

\section{Mass Spectrometry (ESI-TOF-MS/MS)}

Liquid Chromatography (LC)-ESI-MS/MS production scanning via triple-stage quadrupole mass spectrometry designed for the identification and quantification of phytochemical composition of $U$. maritima aqueous extract. The TOF mass analyser displays high mass accuracy ( $\sim 3 \mathrm{ppm})$, confirmation of molecular formulas and high-quality results in the accurate assignment of recovered fragments. A highly purified water extract from U. maritima was analysed by HPLC coupled with ESI-TOF-MS/MS. The HPLC analysis was performed in a Waters 2795 separation module fitted with an auto-injector $(20-\mu \mathrm{L})$ injection loop coupled to aVarian 385-LC evaporative lights scattering detector. Reverse-phase column chromatography analysis was conducted on a C-18 column supplied by Phenomenex Jupiter (USA). The LC-ESI-MS/MS analysis was performed using a Bruker Daltonics micro-OTOF-Q mass spectrometer linked to a Dionex UltiMate 3000 LC.

\section{SH-SY5Y Neuroblastoma Cell Culture}

The human malignant neuroblastoma SH-SY5Y cell line was kindly provided by Tissue Culture Research Laboratory, Institute of Science (IOS), University Technology MARA. The SH-SY5Y cells were cultured in 1:1 EMEM: Ham's F12 nutrient mixture (Sigma, USA) with $1 \%$ non-essential amino acids, $1 \%$ Lglutamine (Sigma, USA), 1\% gentamicin $\left(10 \mathrm{mgmL}^{-1}\right)$ and $10 \%$ Fetal Bovine Serum (FBS). The SH-SY5Y cells were maintained in an incubator in a $5 \% \mathrm{CO}_{2}$ atmosphere with $95 \%$ humidity at $37^{\circ} \mathrm{C}$.

\section{$R$ A-Differentiated (Neuron-Model) Cell Culture}

Retinoic acid (Sigma, USA) well known as potentially enhance differentiation condition of the SHSY5Y neuroblastoma cells to neuron phenotype characteristics. Approximately $2 \times 10^{4}$ a density of SHSY5Y neuroblastoma cells per well were plated in a 96well plate. The following day Retinoic acid was added at a final concentration of $10 \mu \mathrm{M}$ in complete culture media of EMEM-F12 supplemented with 1\% glutamine (Sigma, USA), 1\% non-essential amino acid (PAA Laboratories $\mathrm{GmbH}$, Australia), 1\% Gentamicin (10 mg $\mathrm{mL}^{-1}$ ) (PAA Laboratories $\mathrm{GmbH}$, Austria) and 10\% fetal bovine serum (PAA Laboratories $\mathrm{GmbH}$, Austria). The cell incubation in a humidified atmosphere containing $5 \% \mathrm{CO}_{2}$ at $37^{\circ} \mathrm{C}$. The media with fresh Retinoic acid (R.A) was renewed after three days; cells were ready for testing on the sixth day.
Viability Inhibition effect of U. maritima on $\mathrm{SH}-$ SY5Y Neuroblastoma Cells

The viability and proliferation inhibition effect of $U$. maritima aqueous extract was determined MTS assay and a GloMax-Multi Detection System (Promega, USA), with reading at $490 \mathrm{~nm}$. In brief, human malignant neuroblastoma SH-SY5Y cells $\left(1 \times 10^{5}\right.$ cells $\left.\mathrm{mL}^{-1}\right)$ were seeded in 96-well plates and incubated overnight in an incubator in a $5 \% \mathrm{CO}_{2}$ atmosphere with $95 \%$ humidity at $37^{\circ} \mathrm{C}$. Following day, $100 \mu \mathrm{L}$ fresh media containing a serial dilution of aqueous $U$. maritima extract, with final concentrations ranging from $100 \mathrm{pg} \mathrm{mL}^{-1}$ to $1 \mathrm{mg} \mathrm{mL}^{-1}$. The plates were incubated with the extract for 24,48 and $72 \mathrm{~h}$. After the corresponding incubation period, $20 \mu \mathrm{L}$ MTS solution (Sigma, USA) was added and incubated for 2 to $4 \mathrm{~h}$. The results are representative of at least three independent experiments and the percentage of viability was calculated using the following formula:

$$
\% \text { Viability }=\frac{\text { Absorbance of test wells }}{\text { Absorbance of control wells }} \times 100
$$

\section{Nuclear Fragmentation Detection by DAPI Probe Assay}

Both Human neuroblastoma SH-SY5Y and neuron-

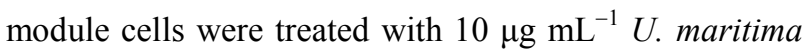
aqueous extract and washed twice with PBS solution after certain incubation times. The cells were fixed with $3.7 \%$ paraformaldehyde, permeabilized for $15 \mathrm{~min}$ and incubated for $10 \mathrm{~min}$ with $1 \mu \mathrm{g} \mathrm{mL} \mathrm{m}^{-1}$ of DNA specific dye (DAPI) The stained cells were imaged (Obj. 40X) in order to examine morphological alteration in the nuclei.

\section{Results}

\section{Characterization Phytochemical Ingredients of Aqueous U. maritima extract}

In the current study, a specific and sensitive method was designed by using ESI-TOF-MS/MS to identify and quantify the major pharmaceutical ingredients of Libyan U. maritima water-based extract. A total of 44 cardiac glycoside compounds and their isomers were characterised. Among these phytochemical compounds, CTSs (namely, bufadienolides) were the most abundant ingredient in the botanical product. Figure 1A indicates that peak retention times (17.585) were further confirmed by HPLC retention times and that the fragmentation pattern was elucidated based on a standardization sample that was identical to proscillaridin A (3-(6-deoxy- $\alpha$-L-mannopyranosyl oxyl14-hydroxybufa-4, 2022 , trienolide, scillarenin $3 \beta$ rhamnoside). The obtained results agree with the standard sample peak for proscillaridin A (SigmaAldrich), as shown in Fig. 1A and 1B. 


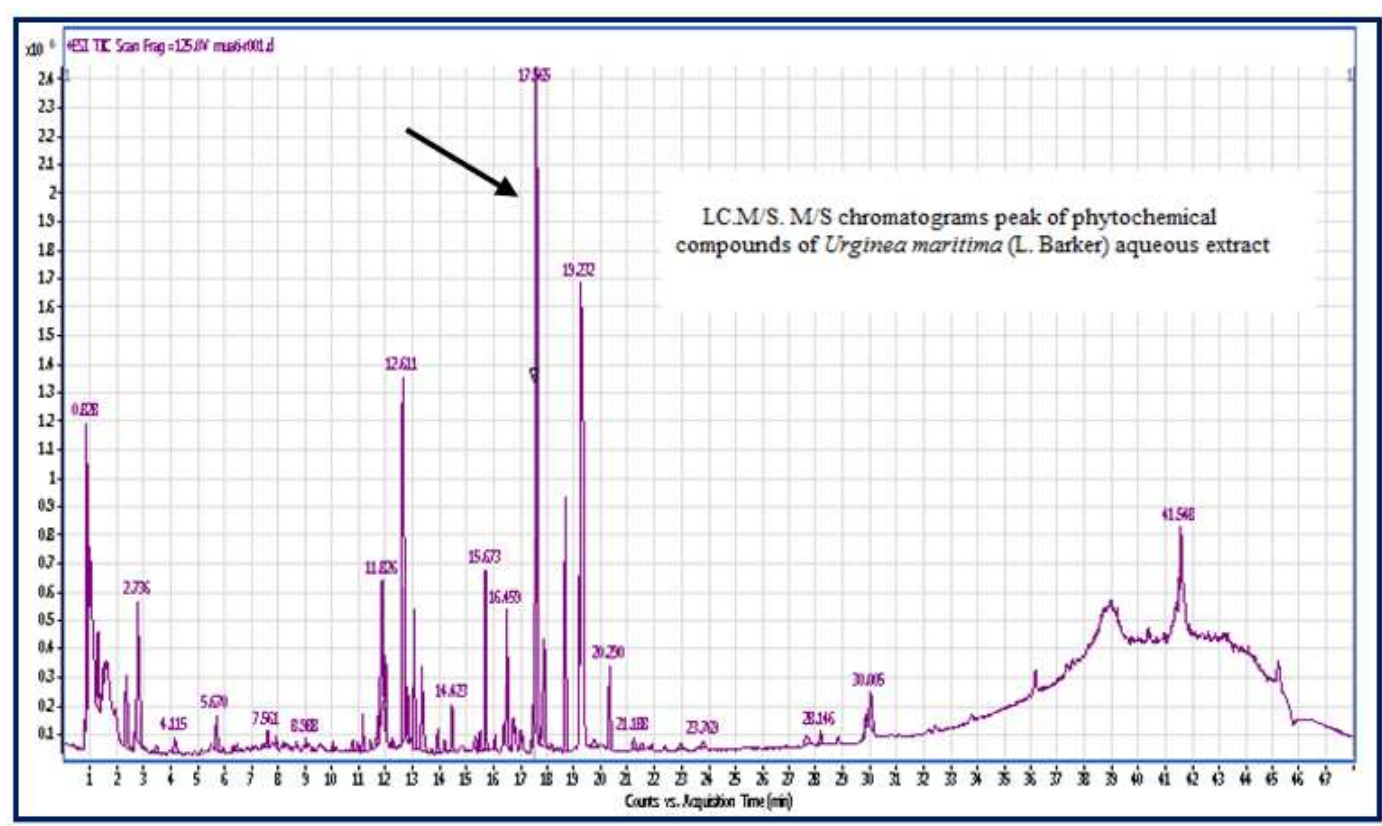

(A)

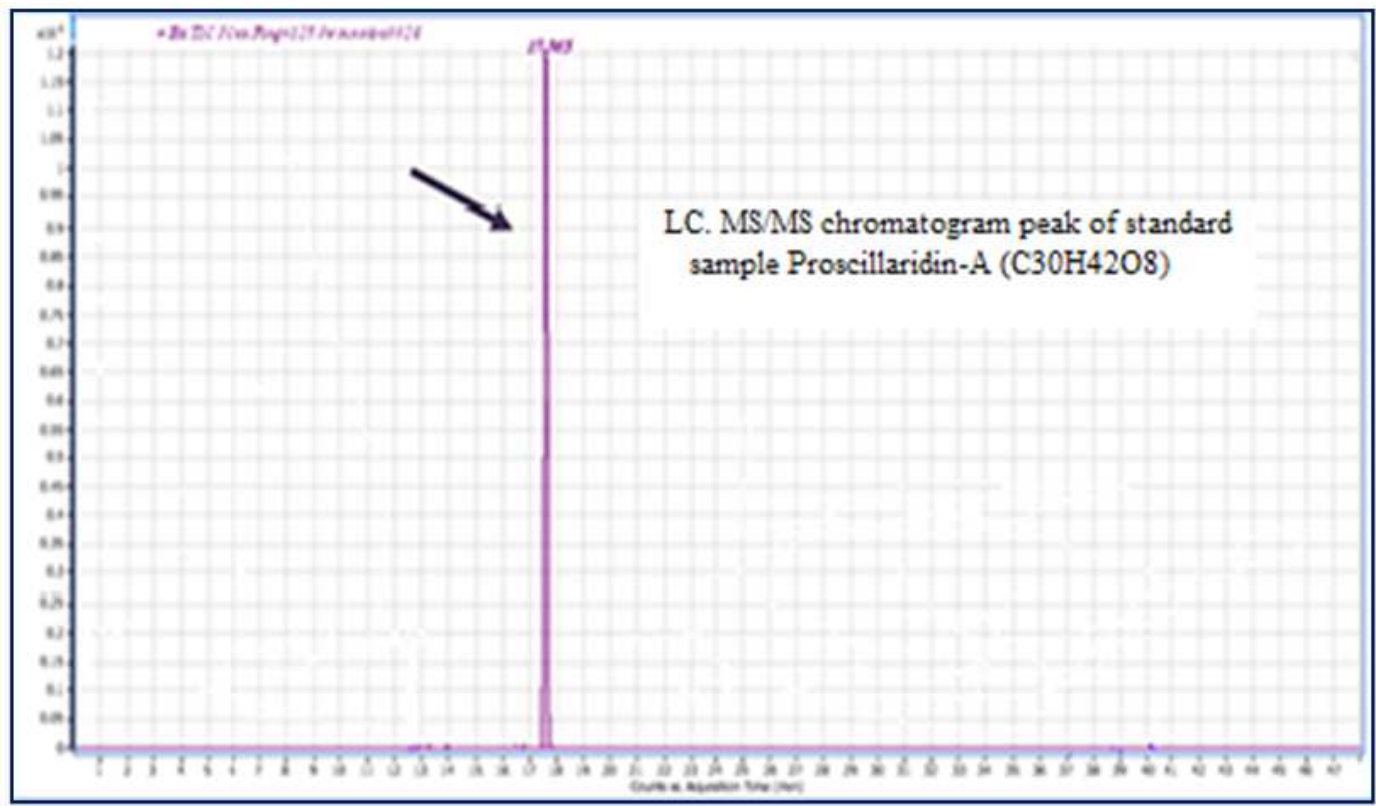

(B)

Fig. 1. (A) LC. M/S M/S chromatograms of Urginea maritima (L. Baker) aqueous extract from (100 $\left.\mu \mathrm{g} \mathrm{mL}^{-1}\right)$, while (B) LC. M/S $\mathrm{M} / \mathrm{S}$ chromatogram peak retention standard sample (Proscillaridin- $\mathrm{A}, \mathrm{C}_{30} \mathrm{H}_{42} \mathrm{O}_{8}$, $10 \mu \mathrm{M}$, obtained data from micro TOF-Q, Analyzed by Agilent Hunte Qualitative Analysis-version B03.01 software

Viability Inhibition Effects of Aqueous U. maritima Extracts

The cell viability of neuroblastoma SH-SY5Y cells was assessed by MTS assay after incubation with different concentration of $U$. maritima water-based extract ranging from $100 \mathrm{pg} \mathrm{mL}^{-1}$ to $1 \mathrm{mg} \mathrm{mL}^{-1}$. The malignant SH-SY5Y neuroblastoma cells exhibited a significant decrease in viability at low U. maritima concentrations, whereby; an eventual decline was noted at the highest concentration of $U$. maritima extract $1 \mathrm{mg}$ $\mathrm{mL}^{-1}$, which it is reported $16 \%$ of the SH-SY5Y cell viability. In relation to that, the estimated $\mathrm{IC}_{50}$ values (concentration that caused the death of $50 \%$ of $\mathrm{SH}$ SY5Y cells) of $U$. maritima aqueous extract were $10 \mu \mathrm{g}$ $\mathrm{mL}^{-1}, 1 \mu \mathrm{g} \mathrm{mL}^{-1}$ and $100 \mathrm{ng} \mathrm{mL} L^{-1}$ after incubation of 24 , 
48 and $72 \mathrm{~h}$, respectively. In contrast, an increased in the total cell numbers among the untreated SH-SY5Y neuroblastoma (negative) control groups was observed. The obtained result display that the action of the viability and proliferation inhibition of the aqueous U. maritima extract increased significantly within a dose-time dependent manner compared with corresponding the untreated SH-SY5Y cells.

\section{Neurotoxic Effects of Aqueous U. maritima Extract}

The incubation of $\mathrm{R}$ A-differentiated cells within a various concentration of $U$. maritima extra consist of 100 pg $\mathrm{mL}^{-1}, 1 \mathrm{ng} \mathrm{mL} \mathrm{m}^{-1}$ and $10 \mathrm{ng} \mathrm{mL}^{-1}$ for $24 \mathrm{~h}$, demonstrated that the RA-differentiated cell viability estimated values $110.58 \% \pm 2.466,111.97 \% \pm 3.399$ and $110.90 \% \pm 6.116$ for the concentration $100 \mathrm{pg} \mathrm{mL}^{-1}, 1 \mathrm{ng}$ $\mathrm{mL}^{-1}$ and $10 \mathrm{ng} \mathrm{mL}^{-1}$ respectively, recorded as the highest percentage of $\mathrm{R}$ A- differentiated cell viability. Particularly, these viability proportions were reported higher than the viability percentage of (negative) control cells. The obtained data demonstrated that nosignificant (ns) neurotoxicity potential of the tested pico and nano-concentrations of $U$. maritima aqueous extract toward the neuronmodel cells. Based on, Oneway ANOVA analysis followed by Dunnett's M.C.T. (Graph Pad Prism 5.01 software).

\section{Results of Nuclear Fragmentation by DAPI Probe}

Malignant neuroblastoma SH-SY5Y cells were examined by fluorescence microscopy (Leica) using $(40 \times s)$ magnification. Late apoptotic and died cells with disrupted cell membranes identified by using the vital dye Propidium Iodide (PI) stain. The nuclei of treated undifferentiated SH-SY5Y cells and RA-differentiated cells were stained with the DNA-specific dye DAPI.

The nuclear morphology indicated by the gradual increase in fluorescence intensity was attributable to high DNA fragmentation. Distinctive apoptotic symptoms appeared after 12, 24 and $48 \mathrm{~h}$ of exposure

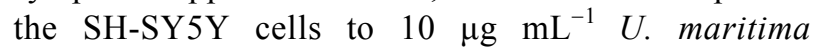
aqueous extract (Fig. 6B).

The nuclear morphology of the control group was rounded, clear-edged and uniformly stained, which was observed in both untreated SH-SY5Y cells and treated neuron-module cells as well. In contrast, apoptotic cells exhibited size-reduced, irregular edges around the nucleus; chromatin concentrated in the nucleus; and an increased number of nuclear body fragments (arrows 6A). The percentage of DNA fragmentation and the formation of apoptotic bodies increased significantly.

\section{Discussion}

Products that more effectively affect the tumorigenic cellular events than those in non- tumorigenic cells are still a critical target that is strongly needed in human neurological disorders. With their great structural diversity, natural products have offered major opportunities for the identification of novel drugs that are active against a wide range of diseases, including neurological disorders (Dunn et al., 2011; Huang et al., 2010; Wen et al., 2014). Recent significant research reported that the mechanisms by which natural extracts are powerful in promoting healing are relatively complex. These mechanisms often regulate a range of genes that affect entire biological processes or cell signaling systems. Conversely, most synthetic drugs address a single biological process or cell signaling pathway. Therefore, natural drugs are suitable for treating an enormous of diseases (Kang et al., 2010; Slingerland et al., 2013). Moreover, a significant number of researchers have become interested in further related investigations, which have sparked the use of CTSs and their natural sources as healthcare and pharmaceutical agents to treat neoplastic cells of diverse histological origin (Mijatovic et al., 2007; Prassas and Diamandis, 2008; Xu et al., 2011).

The term "CTSs" refers to natural, mostly plantderived compounds. There are two classes of CTSs: Namely, cardenolides and bufadienolides. Both classes share the ability to operate as potent inhibitors of the plasma membrane $\mathrm{Na}(+) / \mathrm{K}(+)$-ATPase (Bagrov et al., 2009; Haux et al., 2001). Recently, widespread scientific work both in vitro and in vivo have discovered that in addition to pumping ions, the $\mathrm{Na}(+) / \mathrm{K}(+)$-ATPase plays a role in the regulation of cell growth by stimulating the expression of various genes and organizes cytosolic cascades linked to many cellular events in different tissues (Prassas and Diamandis, 2008). This novel finding suggests that CTSs disrupt important key cellular pathways; hence, CTSs may be considered as a precious pharmaceutical agent in cancer therapeutics (Newman et al., 2008; Slingerland et al., 2013). In this preliminary study, we have focused our interest on the medicinal plant $U$. maritima to identify, quantify and elucidate it's biological properties to discover the effectiveness of this natural compound against human malignant neuroblastoma. The current report is the first to conclude that bufadienolides (namely, Proscillaridin-A) and their isomers as well as a cocktail of polyphenols that are typical of the phytochemical composition of watersoluble extracts of $U$. Maritima (Fig. 1). Extensive studies have revealed that the $\mathrm{Na}(+) / \mathrm{K}(+)$-ATPase, with its highly specific ligands (CTSs), is capable of producing profound anticancer effects, including significant inhibition of proliferation, induction of apoptosis and cell cycle arrest in a large panel of cancer cells (Daniel et al., 2003; Waheed et al., 2012; Zhitu et al., 2012). 


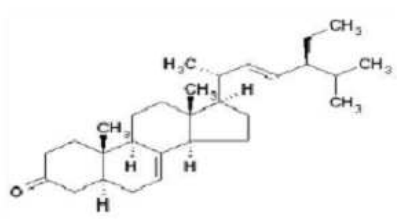

Proscillaridin -A<smiles>COc1cc(-c2oc3cc(O)cc(O)c3c(=O)c2O)ccc1O</smiles>

Kaempferol 3-O-glucoside

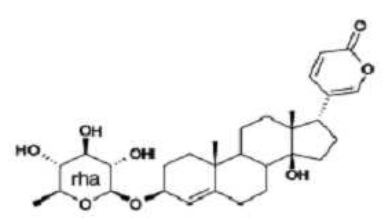

Stigmasterol<smiles>CCC1OC(OCc2oc3cc(O)cc(C)c3c(=O)c2-c2ccc(Cl)cc2)[C@H](C)[C@@H](C)[C@H]1C</smiles>

Gallic acid<smiles>[CH]C1CCC2(C)C(=CCC3C2CCC2(C)C(C(C)CCCC(C)C)CCC32)C1</smiles>

Leucocyanidin<smiles>Oc1cc(O)c2c(c1)O[C@H](c1ccc(O)c(O)c1)[C@H](O)[C@H]2O</smiles>

Fig. 2. The structure of important phytochemical compounds involved in the medicinal herb U. maritima aqueous extract
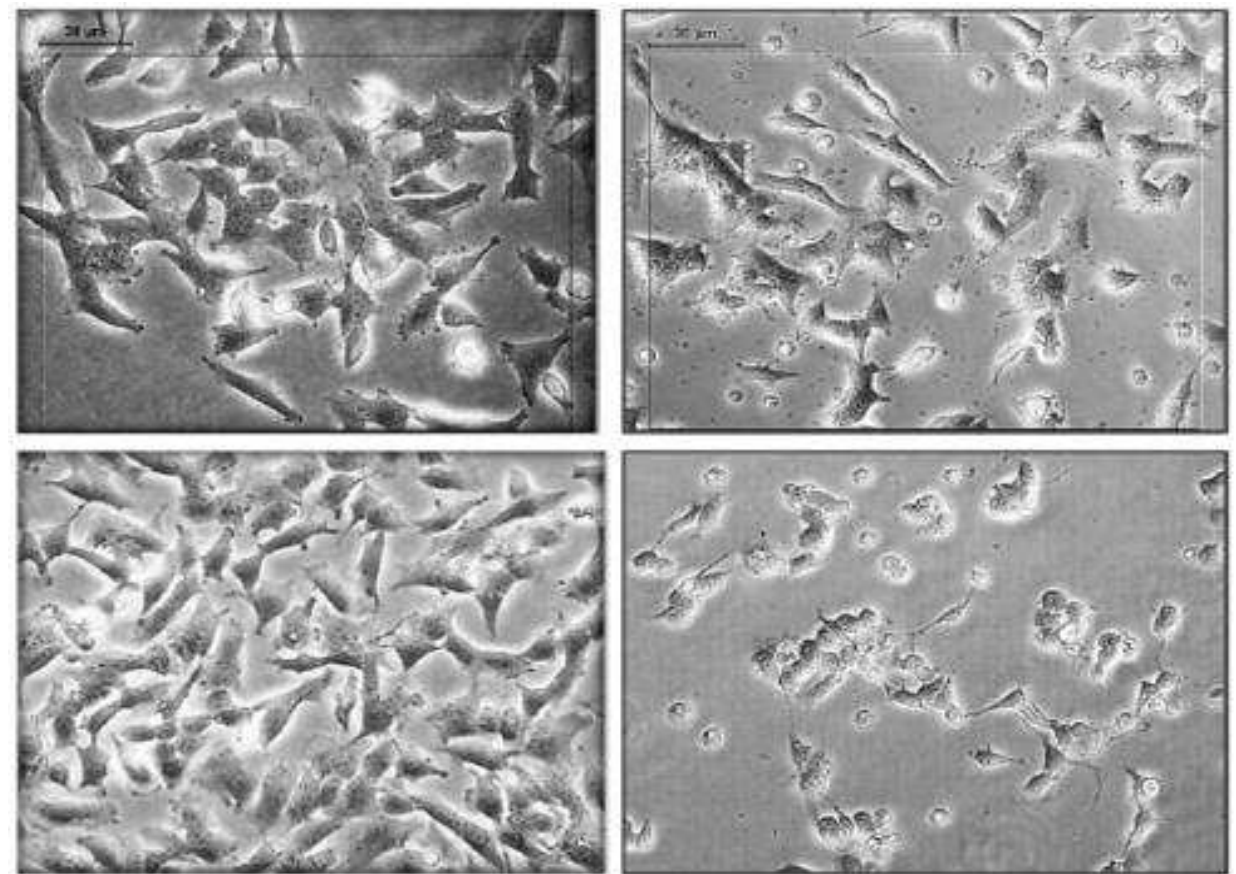

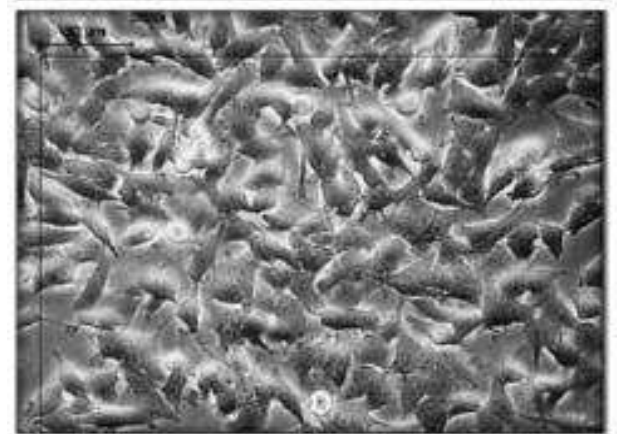

(A)

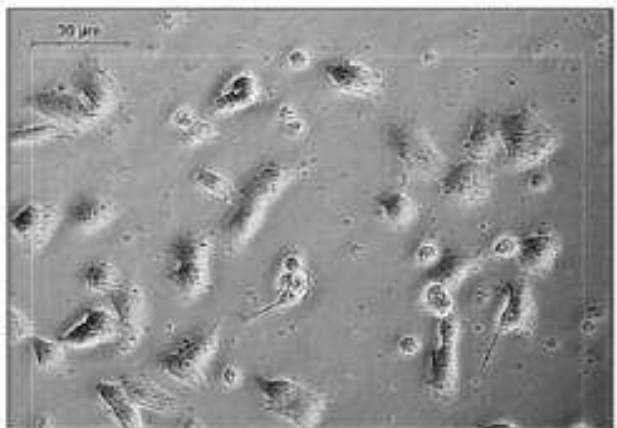

(B)

Fig. 3. Photo represented a morphological alteration of malignant $\mathrm{SH}-\mathrm{SY} 5 \mathrm{Y}$ cells after treating with $\mathrm{IC}_{50}$ of aqueous $U$. maritima extract, at different duration, Cells were observed under Phase contrast inverted Microscopy (Leica, Obj-20 xs) (A) negative control (untreated cells) (B) U. maritima treated SH-SY5Y cells 


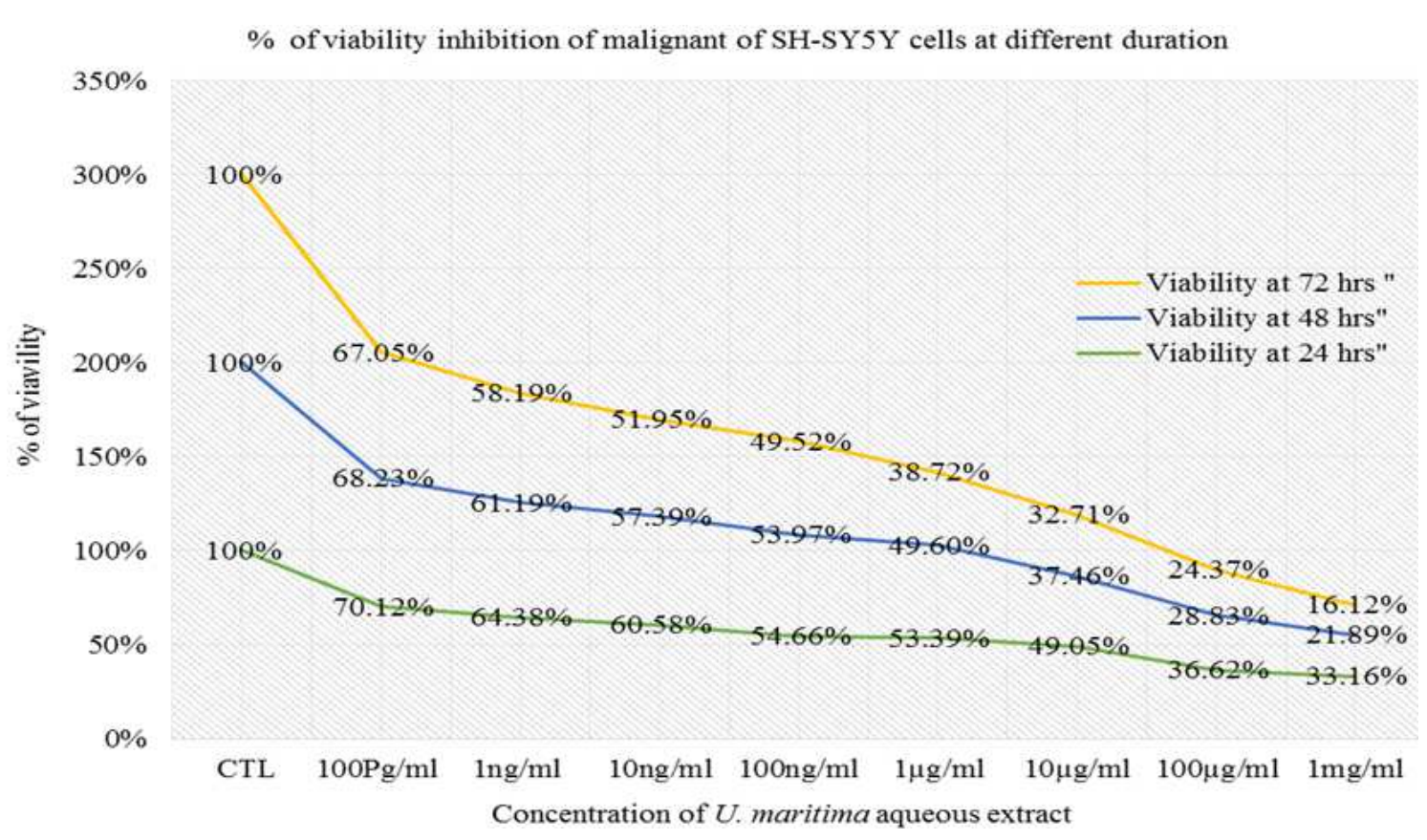

Fig. 4. The diagram representing the mean of the SH-SY5Y neuroblastoma cell viability were shown significantly decreased in a dose-time dependent manner after exposure to various concentrations of $U$. maritima aqueous extract ranging from $100 \mathrm{pg}$ $\mathrm{mL}^{-1}$ to $1 \mathrm{mg} \mathrm{mL}^{-1}$ at different duration 24,48 and $72 \mathrm{~h}$. Compared with corresponding control cells. Each data represents the mean $\pm \mathrm{SD}$ of three independent experiments, $\mathrm{n}=$ nine

A successful study conducted by Prassas and Diamandis (2008) reported that CTSs inhibit the growth of numerous cancer cells, with a threshold concentration around the nanomolar level, which is similar to the therapeutic plasma concentration in patients treated with CTSs (Lopez-Lazaro et al., 2005). Furthermore, interestingly, malignant cells are generally more susceptible to the effects of CTSs than normal cells. This mediation may be caused by the altered density of an enzyme in the plasma cell membrane and by changes in isoenzyme expression in malignant cells (Malikova et al., 2008; Rashan et al., 2011). In line with this observation, the present study's data also showed that the U. maritima composition caused greatly significantly reduced viability coupled with morphological alteration of malignant neuroblastoma SH-SY5Y cells (Fig. 3). With less susceptibility among neuron-like cells upon exposure to a nano-to-microscale concentration of $U$. maritima as confirmed quantitatively by the MTS colorimetric assay (Fig. 4 and 5) and as qualitatively well established by fluorescence imaging using the PI stain (Fig. 6A). In the present study, these characteristics were observed after exposure of SH-SY5Y cells to different concentrations of aqueous U. maritima extract. The apoptotic effect of the botanical extract was attributable to increases in the numbers of both early apoptotic and late apoptotic cells as confirmed by using DNA-specific dye (DAPI). Moreover, chromatin condensation and nuclear fragmentation, which led to profound apoptosis (Fig. 6C). This characteristic highly clear with increasing the time points of incubation of neuroblastoma SH-SY5Y cell with micro-concentration of $U$. maritima aqueous extract, which indicated that apoptosis death mechanism is closely linked to the cytotoxic mechanism of the active constituents containing in the U. Maritima aqueous extract (Fig. 6B).

From a clinical viewpoint, the phytochemical constituents of $U$. maritima are ideal for malignancy treatment because of their preferential killing of tumor cells via apoptosis rather than necrosis; due to the fact that death occurs by apoptosis, the cells break into fragments that are subsequently isolated by phagocytes, hence reducing cellular inflammation. Collectively, these clinical observations, along with the safety profile of CTSs, which have well-known usage in medical cardiology, have established evidence identifying the constituents of $U$. maritima water-based extract as distinctly promising compounds that have the antitumor capability. Advanced studies have emphasized significant results for the cytotoxicity and antiproliferative capability of CTSs (such as bufadienolides), Proscillaridin-A, Stigmasterol, Spinasterol and 6-stigmasten-3beta in various human cancer cell lines (Dunn et al., 2011; Winnicka et al., 2010). On the other hand, several investigations have confirmed that Stigmasterol, Spinasterol, 6-stigmasten3 beta compounds, in addition to well-known natural antioxidants that include Quercetin, Kaempferol, 
Leucocyanidin and Gallic acid, are cytoprotective and neuroprotective in a neuron model (Choi et al., 2012; Kelsey et al., 2011; Oselkin et al., 2010). Other compelling in vivo anti-inflammatory study performed by Wen et al. (2014) revealed that bufalin significantly downregulated the expression levels of proinflammatory signals, including Nitric Oxide Synthase (iNOS), cyclooxygenase-2 (COX-2), IL-1 $\beta$, IL-6 and
TNF- $\alpha$. This interesting study provides evidence that bufalin possesses strong anti-inflammatory action in vivo, which may involve reduced activation of $\mathrm{NF}-\kappa \mathrm{B}$ and suppression of pro-inflammatory mediators. Along with previous results observed in various other preclinical investigations, these findings confirm the idea that bufadienolides could be a novel potential therapeutic agent for the treatment of inflammatory diseases.
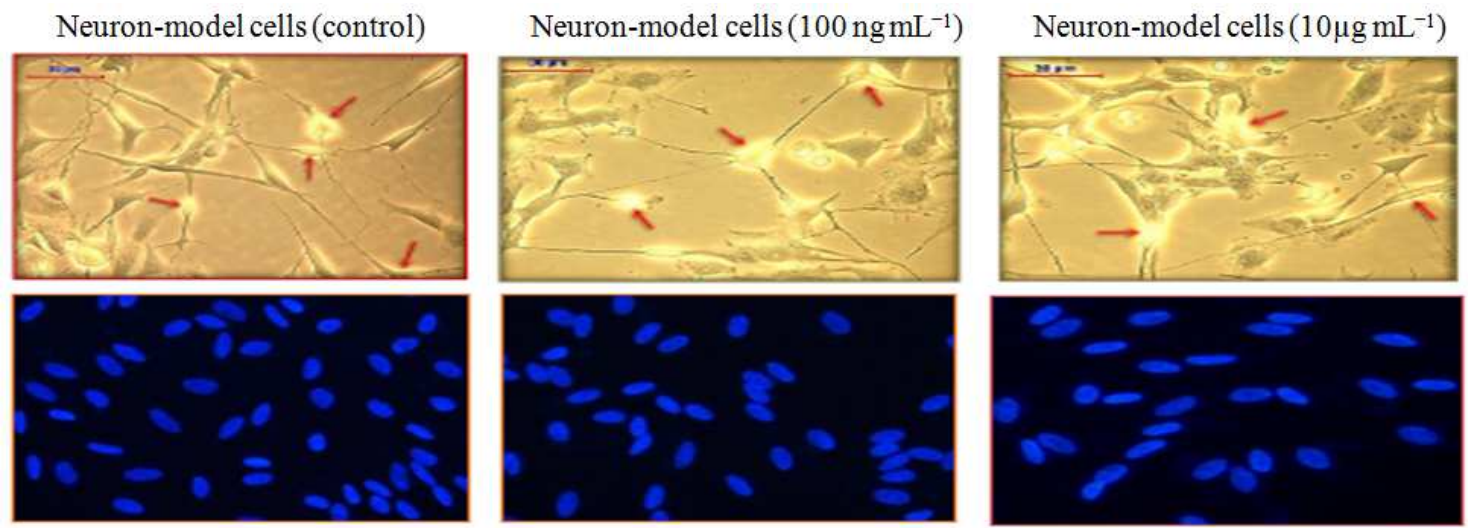

(A)

Neurotoxicity effect of $U$. maritima extract on neuron-module cells

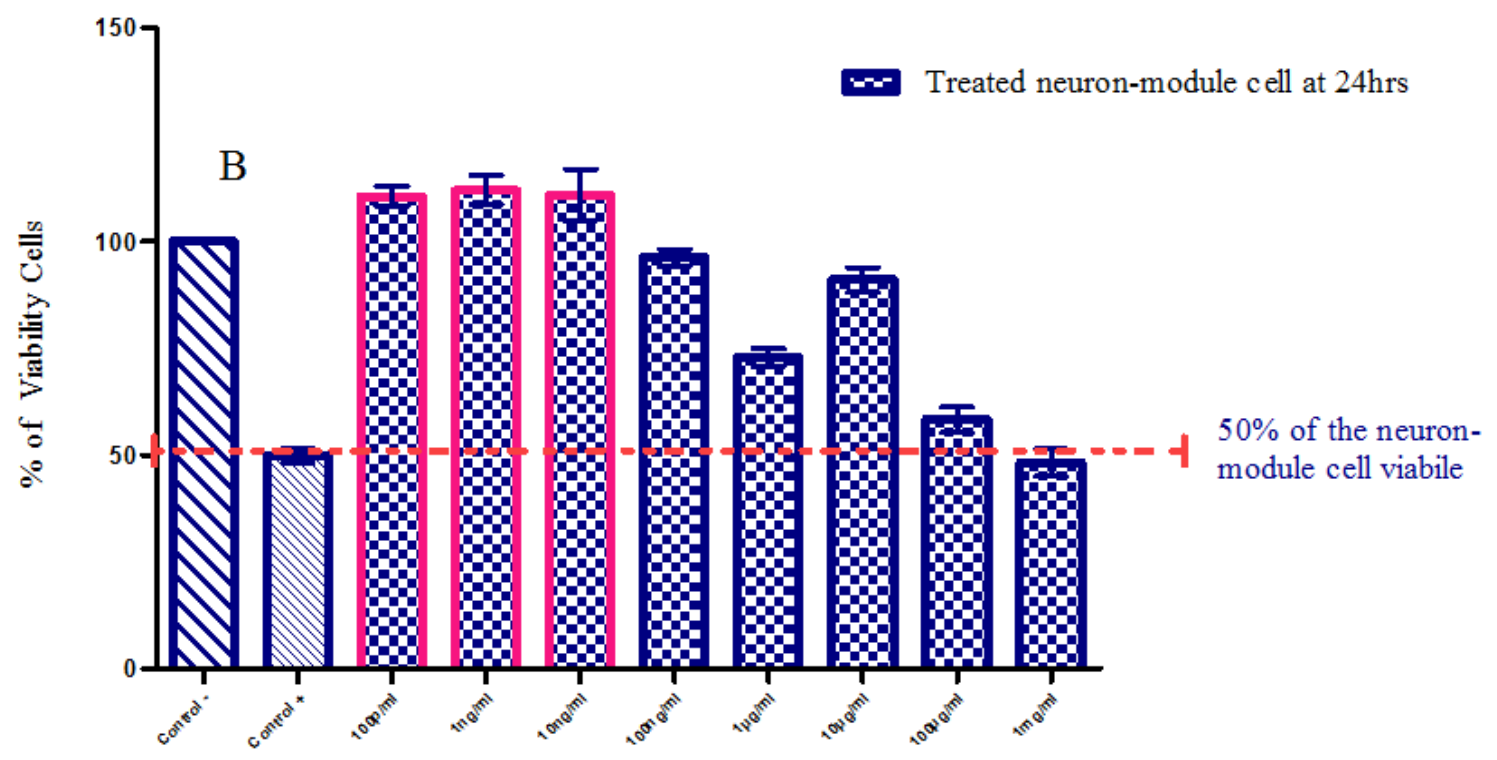

Various Concentration of $U$. maritima aqueous extract

(B)

Fig. 5. The Representative photos show non-significant (ns) neurotoxicity activities of aqueous U. maritima extract on neuronmodule cell after exposure to testing IC50 concentrationsof $U$. maritima compared to negative control (untreated) neuronmodule cells (A), at duration $24 \mathrm{~h}$. Cells observed under phase contrast inverted fluorescent microscope (Leica, Obj-20 xs). Chart represented the mean percentage of neurotoxicity of $U$. maritima on neuron-model cell compares with the control group (a) were shown non-significant (ns) neurotoxicity bioactivity of most tested doses of aqueous U. maritima extract, that ranging from $100 \mathrm{pg} \mathrm{mL}^{-1}$ to $1 \mathrm{mg} \mathrm{mL}^{-1}$. Red column showed extend of neuron-module cells viability more than the untreated cells (negative) control at duration. Each data represents the mean $\pm \mathrm{SD}$ of three independent experiments, $\mathrm{n}=$ nine 


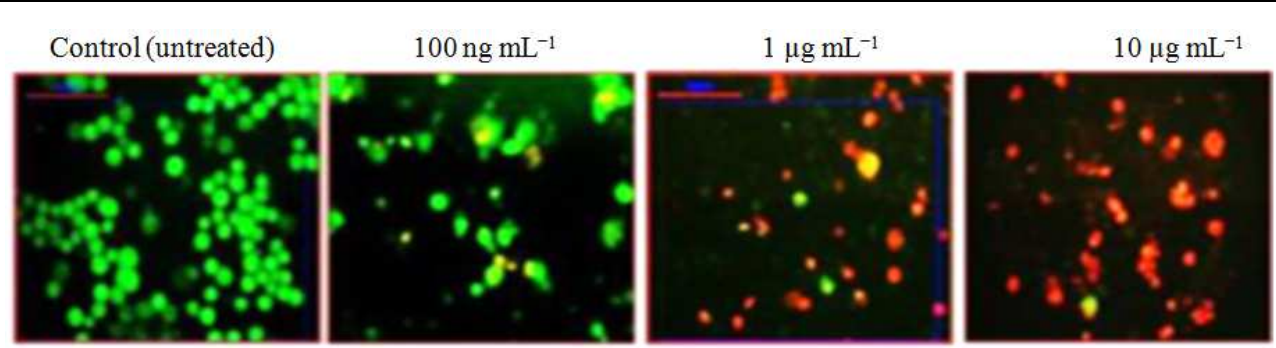

(A)

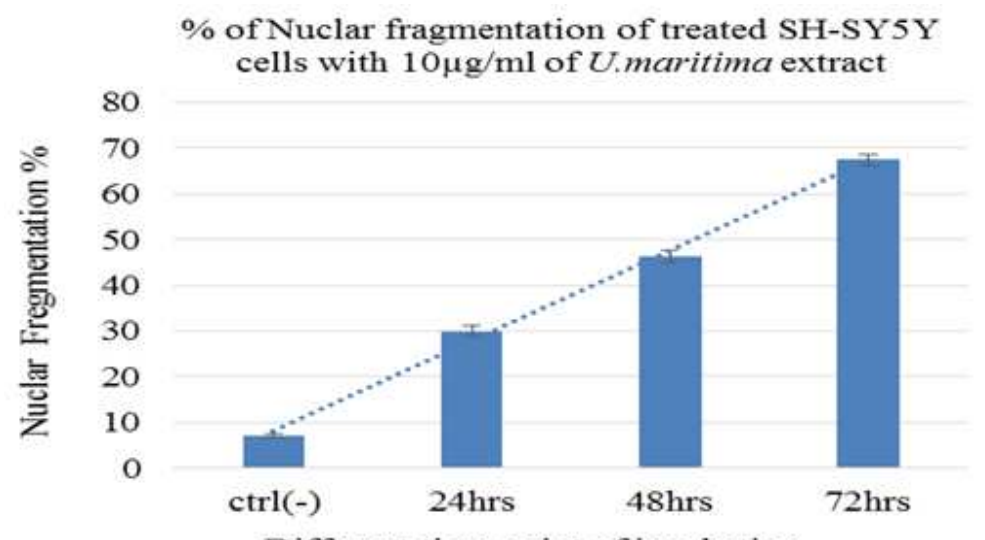

Different time point of incubation

(B)
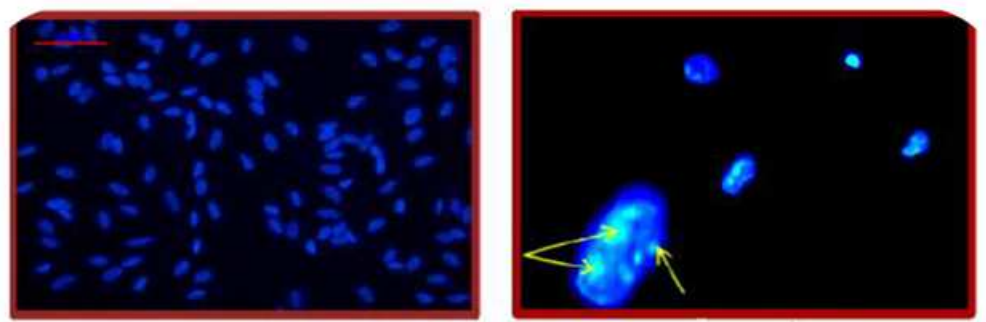

Corl (untreated) SH-SY5Y cell line
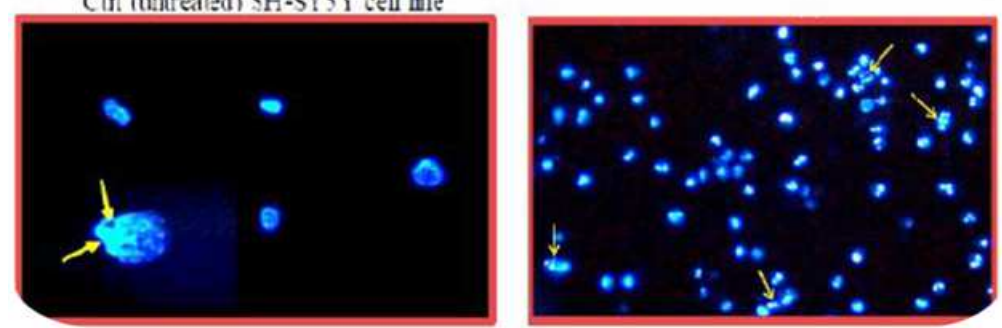

(C)

Fig. 6. (A) Both treated and untreated human malignant neuroblastoma SH-SY5Y cells were stained with PI staining. Cells were observed under a fluorescence microscope (Leica $\times 20$ ). Viable cells show green fluorescence in control (untreated) cells. Early and late and apoptotic cells show yellow and orange fluorescence in the treated groups. Concurrently, dead neuroblastoma has red fluorescence after time point $24 \mathrm{~h}$ of incubation; (B) The chart represented the proportion of nuclear fragmentations of DAPI-stained SH-SY5Y cells. The finding indicates that effects of phytochemical constituents of aqueous U. maritima extract as efficient apoptosis inducer depends on time points, data are presented as mean \pm SD; (C) SH-SY5Y cells by fluorescence inverted microscope (Leica) using $40 \times$ magnification. The nuclei were stained with the DNA-specific dye (DAPI) after exposure to $10 \mu \mathrm{g} \mathrm{ml}^{-1}$ of $U$. maritima aqueous extracts apoptotic distinctive symptoms appeared after $10 \mathrm{~h}$ exposure. Treated cells exhibiting size-reduced nuclear and plasma membrane blebbing and chromatin condensed (the arrows). DNA fragmentation and the formation of apoptotic bodies were observed (the arrows), compared to the nuclear morphology of cells control (untreated) group, had a regular and an oval shape and uniformly stained. Each photograph was representative of three independent observations 
Furthermore, remarkable searches suggest that combinations of numerous antioxidants with antiinflammatory agents may be more powerful in prevention and much more promising as a strategic approach to combat the neurological disorders (Lee and Lee, 2007; Deng et al., 2011; Wen et al., 2014). The aforementioned suggestion may explain how the presence of a combination of the bufadienolides and a cocktail of polyphenolic compounds (Fig. 2), as the natural principal phytochemical ingredients of $U$. maritima water-based extractprovides significant selective proliferation inhibition and the effective apoptosis-inducing activities against human malignant neuroblastoma with non-significant neurotoxicity toward RA-differentiated cells (neuron-like cells), in vitro.

\section{Conclusion}

Tumor specificity is one of the biggest challenges facing new anticancer pharmaceutical candidates. The present investigation illustrates that the phytochemical composition of $U$. maritima extract meets the fundamental criteria for an effective agent against the human neuroblastoma SH-SY5Y cell line, with less sensitivity among neuron-module cells, In vitro. Deeper investigation should follow this work to elucidate the molecular mechanism of the anticancer activity of this unusual class of natural products, which could produce new structures in drug design to combat human malignant neuroblastoma in particular and neurological disorders in general.

\section{Acknowledgement}

For the research work, the Faculty of Applied Science, and Tissue Culture Research Laboratory, Institute of Science (IOS), University Technology Mara (UiTM), Shah Alam, Malaysia, would be appreciated for their effective approach in timely provision of facilities and services.

\section{Author's Contributions}

All authors equally contributed in this work.

\section{Ethics}

This article is original and contains unpublished material. The corresponding author confirms that all of the other authors have read and approved the manuscript and no ethical issues involved.

\section{References}

Bagrov, A.Y., J.I. Shapiro and O.V. Fedorova, 2009. Endogenous cardiotonic steroids: Physiology, pharmacology and novel therapeutic targets. Pharmacol. Rev., 61: 9-38.

DOI: $10.1124 /$ pr.108.000711
AL-Tardeh, S.T.S. and B.E.D. Delivopouos, 2006. Anatomical studies on the adventitious roots of the geophyte Urginea maritima (L.) baker. J. Biol. Res., 5: 61-70.

Blaylock, R.L. and J. Maroon, 2012. Natural plant products and extracts that reduce immunoexcitotoxicityassociated neurodegeneration and promote repair within the central nervous system. Surgical Neurol. Int., 3: 19-19. PMID: 22439110

Cernaianu, G., P. Brandmaier, G. Scholz, O.P. Ackermann and R. Alt et al., 2008. All-trans retinoic acid arrests neuroblastoma cells in a dormant state. Subsequent nerve growth factor/brain-derived neurotrophic factor treatment adds modest benefit. J. Pediatric Surgery, 43: 1284-1294. DOI: $10.1016 /$ j.jpedsurg.2008.01.007

Choi, D.Y., Y.J. Lee, J.T. Hong and H.J. Lee, 2012. Antioxidant properties of natural polyphenols and their therapeutic potentials for Alzheimer's disease. Brain Res. Bull., 87: 144-153.

DOI: 10.1016/j.brainresbull.2011.11.014

Daniel, D., C. Susal, B. Kopp, G. Opelz and P. Terness, 2003. Apoptosis-mediated selective killing of malignant cells by cardiac steroids: Maintenance of cytotoxicity and loss of cardiac activity of chemically modified derivatives. Int. Immunopharmacol., 3: 1791-1801. DOI: 10.1016/j.intimp.2003.08.004

Deng, J.S., C.S. Chi, S.S. Huang, P.H. Shie and T.H. Lin et al., 2011. Antioxidant, analgesic and antiinflammatory activities of the ethanolic extracts of Taxillus liquidambaricola. J. Ethnopharmacol., 137: 1161-1171.DOI: 10.1016/j.jep.2011.07.041

Dunn, D.E., D.N. He, P. Yang, M. Johansen and R.A. Newman et al., 2011. In vitro and in vivo neuroprotective activity of the cardiac glycoside oleandrin from Nerium oleander in brain slice-based stroke models. J. Neurochem., 119: 805-814. DOI: $10.1111 /$ j.1471-4159.2011.07439.x

EL-Seedi, H.R., R. Burman, A. Mansour, Z. Turki and L. Boulos et al., 2013. The traditional medical uses and cytotoxic activities of sixty-one Egyptian plants: Discovery of an active cardiac glycoside from Urginea maritima. J. Ethnopharmacol., 145: 746-757. DOI: $10.1016 /$ j.jep.2012.12.007

Felth, J., L. Rickardson, J. Rosen, M. Wickstrom and M. Fryknas et al., 2009. Cytotoxic effects of cardiac glycosides in colon cancer cells, alone and in combination with standard chemotherapeutic drugs. J. Natural Product, 72: 1969-1974. DOI: $10.1021 / \mathrm{np} 900210 \mathrm{~m}$

Haux, J., O. Klepp, O. Spigset and S. Tretli, 2001. Digitoxin medication and cancer; case control and internal dose-response studies. BMC Cancer, 1: 11-11. DOI: $10.1186 / 1471-2407-1-11$ 
Huang, W.Y., Y.Z. Cai and Y. Zhang, 2010. Natural phenolic compounds from medicinal herbs and dietary plants: Potential use for cancer prevention. Nutr. Cancer, 62: 1-20. DOI: 10.1080/01635580903191585

Jiang, Y., Y. Zhang, J. Luan, H. Duan and F. Zhang et al., 2010. Effects of bufalin on the proliferation of human lung cancer cells and its molecular mechanisms of action. Cytotechnology, 62: 573-583. DOI: $10.1007 / \mathrm{s} 10616-010-9310-0$

Kang, K.A., Z.H. Wang, R. Zhang, M.J.Piao and K.C. Kim et al., 2010. Myricetin protects cells against oxidative stress-induced apoptosis via regulation of PI3K/Akt and MAPK signaling pathways. Int. J. Molecular Sci., 11: 4348-4360. DOI: $10.3390 /$ ijms 11114348

Kelsey, N., W. Hulick, A. Winter, E. Ross and D. Linseman, 2011. Neuroprotective effects of anthocyanins on apoptosis induced by mitochondrial oxidative stress. Nutrit. Neurosci., 14: 249-259. DOI: 10.1179/1476830511Y.0000000020

Kopp, B., L. Krenn, M. Draxler, A. Hoyer and R. Terkola et al., 1996. Bufadienolides from Urginea maritima from Egypt. Phytochemistry, 42: 513-522. DOI: 10.1016/0031-9422(95)00876-4

Lee, S.J. and K.W. Lee, 2007. Protective effect of (-)epigallocatechin gallate against advanced glycation endproducts-induced injury in neuronal cells. Biol. Pharmaceutical Bull., 30: 1369-1373.

DOI: $10.1248 / \mathrm{bpb} .30 .1369$

Lopez-Lazaro, M., N. Pastor, S.S. Azrak, M.J. Ayuso and C.A. Austin et al., 2005. Digitoxin inhibits the growth of cancer cell lines at concentrations commonly found in cardiac patients. J. Nat. Prod., 68: 1642-1645. PMID: 16309315

Malikova, J., J. Swaczynova, Z. Kolar and M. Strnad, 2008. Anticancer and antiproliferative activity of natural brassinosteroids. Phytochemistry, 69: 418-426. DOI: $10.1016 /$ j.phytochem.2007.07.028

Mijatovic, T. and R. Kiss, 2013. Cardiotonic steroidsmediated $\mathrm{Na}+/ \mathrm{K}+$-ATPase targeting could circumvent various chemoresistance pathways. Planta Med., 79: 189-198. DOI: $10.1055 / \mathrm{s}-0032-1328243$

Mijatovic, T., E. Van Quaquebeke, B. Delest, O. Debeir and F. Darro et al., 2007. Cardiotonic steroids on the road to anti-cancer therapy. Biochimica et Biophysica Acta (BBA)-Rev. Cancer, 1776: 32-57. DOI: 10.1016/j.bbcan.2007.06.002

Newman, R.A., P. Yang, A.D. Pawlus and K.I. Block, 2008. Cardiac glycosides as novel cancer therapeutic agents. Molecular Intervent., 8: 36-36. DOI: $10.1124 / \mathrm{mi} .8 .1 .8$

Oselkin, M., D. Tian and P.J. Bergold, 2010. Low-dose cardiotonic steroids increase sodium-potassium ATPase activity that protects hippocampal slice cultures from. Neurosci. Lett., 473: 67-71.

DOI: 10.1016/j.neulet.2009.10.021
Park, J.R., A. Eggert and H. Caron, 2010. Neuroblastoma: Biology, prognosis and treatment. Hematol. Oncol. Clinics North Am., 24: 65-86. DOI: 10.1016/j.hoc.2009.11.011

Prassas, I. and E. Diamandis, 2008. Novel therapeutic applications of cardiac glycosides. Nature Rev. Drug Discovery, 7: 926-935. DOI: 10.1038/nrd2682

Rashan, L.J., K. Franke, M.M. Khine, G. Kelter and H.H. Fiebig et al., 2011. Characterization of the anticancer properties of monoglycosidic cardenolides isolated from Nerium oleander and Streptocaulon tomentosum. J. Ethnopharmacol., 134: 781-788. DOI: 10.1016/j.jep.2011.01.038

Slingerland, M., C. Cerella, H.J. Guchelaar, M. Diederich and H. Gelderblom, 2013. Cardiac glycosides in cancer therapy: From preclinical investigations towards clinical trials. Investigat. New Drugs, 31: 1087-1094. DOI: 10.1007/s10637-013-9984-1

Waheed, A., J. Barker, S.J. Barton, C.P. Owen and S. Ahmed et al., 2012. A novel steroidal saponin glycoside from Fagonia indica induces cellselective apoptosis or necrosis in cancer cells. Eur. J. Pharmaceutical Sci., 47: 464-473. DOI: $10.1016 /$ j.ejps.2012.07.004

Wang, X.M., J.B. Plomley, R.A. Newman and A. Cisneros, 2000. LC/MS/MS analyses of an oleander extract for cancer treatment. Analytical Chem., 72: 3547-3552. DOI: 10.1021/ac991425a

Wen, L., Y. Huang, X. Xie, W. Huang and J. Yin et al., 2014. Anti-inflammatory and antinociceptive activities of bufalin in rodents. Mediators Inflammat. DOI: 10.1155/2014/171839

Winnicka, K., K. Bielawski, A. Bielawska and W. Miltyk, 2010. Dual effects of ouabain, digoxin and proscillaridin $\mathrm{A}$ on the regulation of apoptosis in human fibroblasts. Natural Product Res., 24: 274-285. DOI: 10.1080/14786410902991878

Xu, Z.W., F.M. Wang, M.J. Gao, X.Y. Chen and N.N. Shan, 2011. Cardiotonic steroids attenuate ERK phosphorylation and generate cell cycle arrest to block human hepatoma cell growth. J. Steroid Biochem. Molecular Biol., 125: 181-191. DOI: 10.1016/j.jsbmb.2010.12.016

Yu, S.P., 2003. $\mathrm{Na}^{+}, \mathrm{K}^{+}$-ATPase: The new face of an old player in pathogenesis and apoptotic/hybrid cell death. Biochem. Pharmacol., 66: 1601-1609.

DOI: 10.1016/S0006-2952(03)00531-8

Zhitu, Z., S. Hongzhi, M. Guangyou, W. Zhenghua and L. Enze et al., 2012. Bufalin induces lung cancer cell apoptosis via the inhibition of PI3K/Akt pathway. Int. J. Mol. Sci., 13: 2025-2035. DOI: $10.3390 /$ ijms 13022025 